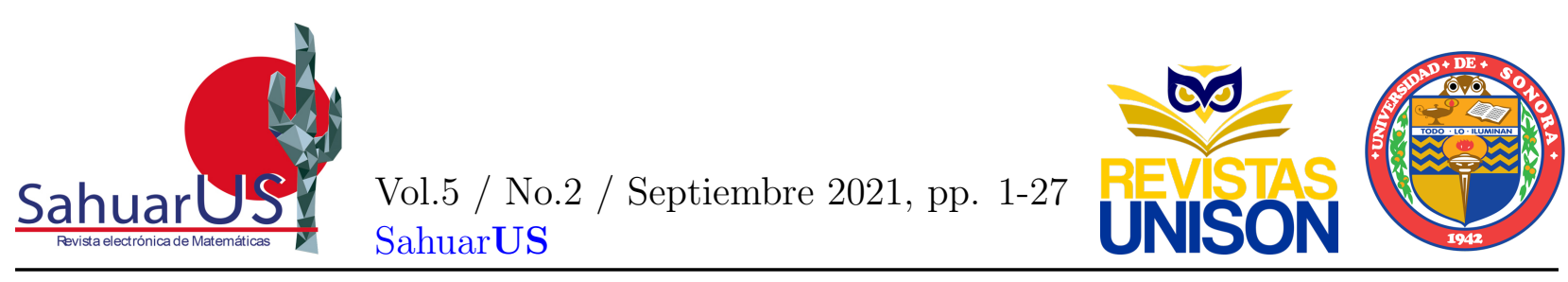

\title{
Un recorrido por el concepto de curvatura
}

\author{
José Luis Cisneros Molina ${ }^{1}$ \\ Instituto de Matemáticas, Unidad Cuernavaca, Universidad Nacional Autónoma de México \\ 1jlcisneros@im.unam.mx
}

\section{Resumen}

La curvatura es uno de los conceptos más importantes en geometría diferencial. En este artículo daremos un recorrido por las ideas detrás de este concepto. Veremos diferentes maneras de definir la curvatura de curvas y diferentes tipos de curvaturas para superficies $y$ variedades de dimensiones superiores.

Palabras Clave: Curvatura normal, curvaturas principales, curvatura Gaussiana, curvatura media, curvatura seccional, curvatura escalar, Teorema Egregio de Gauss, Teorema de Gauss-Bonnet.

DOI: $10.36788 /$ sah.v5i2.120

Recibido: 8 de julio de 2021.

Aceptado: 14 de septiembre de 2021.

\section{Introducción}

El objetivo de este artículo es presentar diferentes nociones de curvatura en geometría diferencial a partir de su significado geométrico en ejemplos, así como la expresión analítica de dichos conceptos. Es una introducción a la geometría diferencial, dirigido a estudiantes de ciencias e ingenierías quienes hayan cursado cálculo de varias variables. Comenzando con la idea intuitiva de que una línea recta no se curva, y de que una circunferencia de radio pequeño se curva más que una circunferencia de radio mayor, se definirá de manera geométrica y analítica la curvatura de curvas planas y en el espacio. Posteriormente, usando la curvatura de curvas planas, introduciremos las curvaturas normales de una superficie, y entre ellas, especificaremos a las curvaturas principales. A partir de éstas últimas, presentaremos la curvatura Gaussiana y la curvatura media de una superficie. Definiremos curvaturas análogas para el caso de hipersuperficies y finalmente definiremos la curvatura seccional y la curvatura escalar de una variedad Riemanniana. Terminamos enunciando algunos teoremas clásicos que involucran a las curvaturas Gaussiana y media de una superficie. Dado que nuestro objetivo es concentrarnos en el aspecto geométrico de la curvatura, no mencionaremos nada acerca de su desarrollo histórico, lo cual también es un tema apasionante,para ello recomendamos el artículo (Naveira, 2007). 


\section{Motivación}

Podemos comprobar empíricamente que a partir de una hoja de papel se pueden formar un cono o un cilindro. Pero es imposible obtener un pedazo de esfera, sin doblar, estirar o cortar la hoja. La razón de esto es que un plano, un cono y un cilindro tienen la misma "curvatura Gaussiana", mientras que en un plano y una esfera dicha curvatura es diferente. Es por esto que es imposible construir el mapa perfecto, es decir, al representar a la superficie de la Tierra en un plano, siempre habrá distorsiones en las distancias. Es posible construir mapas que preserven los ángulos de las trayectorias recorridas, como el Mapa de Mercator, o mapas que preserven las áreas, pero no se puede construir un mapa que preserve todas estas propiedades, ni que preserve todas las distancias ${ }^{1}$

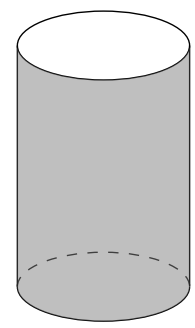

(a) Cilindro

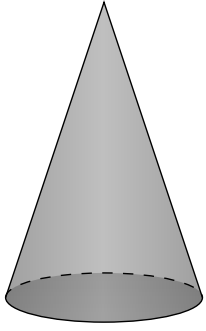

(b) Cono

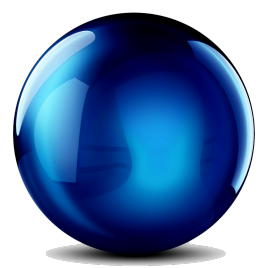

(c) Esfera

\section{Curvas en el plano}

Comenzaremos considerando curvas en el plano Euclidiano $\mathbb{R}^{2}$. Recordemos que $\mathbb{R}^{2}$ consiste de todas las parejas ordenadas de números reales $\mathbb{R}^{2}=\{(x, y) \mid x, y \in \mathbb{R}\}$.

Sea $I=[0,1]$. Una función $\alpha: I \rightarrow \mathbb{R}^{2}$ es de la forma $\alpha(t)=(x(t), y(t))$, donde las funciones $x: \mathbb{R} \rightarrow \mathbb{R}$ y $y: \mathbb{R} \rightarrow \mathbb{R}$ son llamadas las funciones coordenadas de $\alpha$. La función $\alpha$ es diferenciable cuando todas sus funciones coordenadas son diferenciables.

Una curva parametrizada diferenciable en $\mathbb{R}^{2}$ es una función diferenciable $\alpha: I \rightarrow \mathbb{R}^{2}$. A su imagen $\alpha(I) \subset \mathbb{R}^{2}$ se le denomina la traza de $\alpha$. Podemos pensar a una curva parametrizada como una partícula moviéndose a lo largo de la traza de $\alpha$, tal que al tiempo $t$ se encuentra en el punto $\alpha(t)$. El vector tangente o vector velocidad de la curva $\alpha$ en el punto $\alpha(t)$, denotado por $\alpha^{\prime}(t)$, es el vector dado por las derivadas de las funciones coordenadas, es decir $\alpha^{\prime}(t)=\left(x^{\prime}(t), y^{\prime}(t)\right)$. Una curva parametrizada diferenciable es regular si el vector tangente es distinto del vector cero para toda $t \in I$.

Dadas una función diferenciable $h: I \rightarrow I$ tal que $h^{\prime}(t)>0$ para toda $t \in I$ y una curva parametrizada diferenciable regular $\alpha: I \rightarrow \mathbb{R}^{2}$, la composición $\beta(t)=\alpha(h(t))$ es otra curva parametrizada regular. Decimos que $\beta$ es una reparametrización de $\alpha$.

\footnotetext{
${ }^{1}$ Para saber más sobre las matemáticas involucradas al hacer mapas, recomiendo el video Mapas, de la serie Aventuras Matemáticas del Instituto de Matemáticas de la UNAM https://www.matem.unam.mx/ $\sim$ video/mapas/map.html
} 


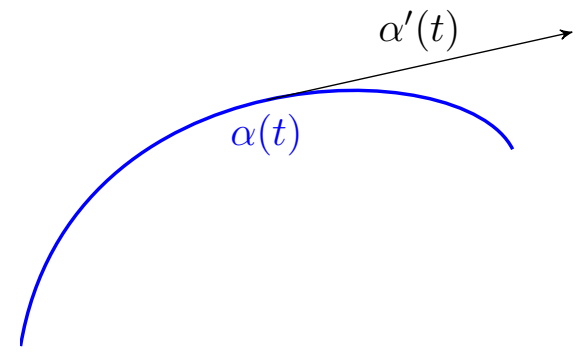

Figura 1: Curva parametrizada y vector tangente.

Dada una curva parametrizada diferenciable regular $\alpha: \mathbb{R} \rightarrow \mathbb{R}^{2}$, siempre es posible encontrar una reparametrización $\beta$ tal que la norma del vector velocidad es 1 en todo punto, es decir, $\left\|\beta^{\prime}(t)\right\|=1$ para toda $t \in I$ (ver (do Carmo, 1994, §1.5, Observación 2)). En este caso decimos que $\beta$ está parametrizada por longitud de arco, ya que el parámetro nos da la longitud recorrida.

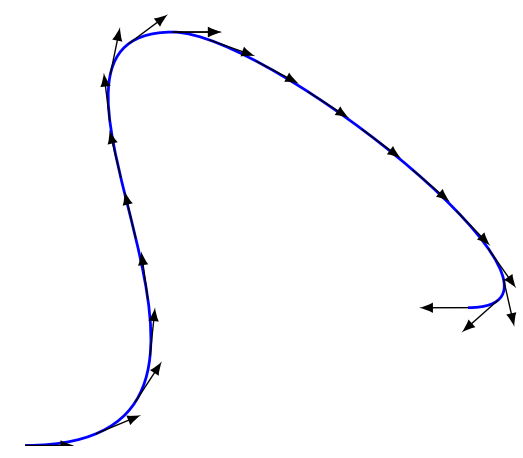

Figura 2: Curva parametrizada por longitud de arco.

\subsection{Curvatura}

El problema que nos planteamos es el siguiente:

Dada una curva parametrizada diferenciable regular $\alpha: I \rightarrow \mathbb{R}^{2}$,

¿Cómo podemos medir qué tanto se curva?

Daremos dos respuestas, una geométrica y una analítica.

\section{Respuesta geométrica}

Partimos de dos ideas intuitivas: 
1. Una recta no se curva. Entonces diremos que una recta tiene curvatura cero.

2. Sean $C_{r_{1}}$ y $C_{r_{2}}$ dos circunferencias de radios $r_{1}$ y $r_{2}$ respectivamente. Si $r_{1}<r_{2}$, entonces $C_{r_{1}}$ se curva más que $C_{r_{2}}$.

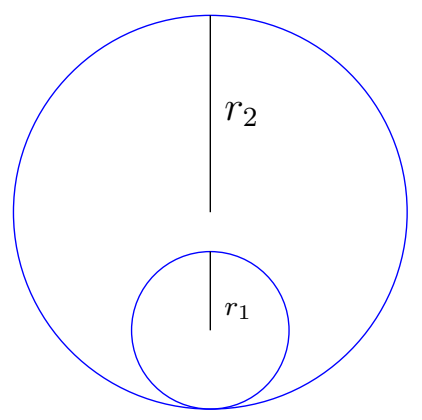

Es decir, la curvatura de una circunferencia es inversamente proporcional a su radio. Entonces diremos que la curvatura de la circunferencia $C_{r}$ de radio $r$ es $\frac{1}{r}$. Esto es compatible con el inciso 1, si consideramos a las rectas como circunferencias de radio infinito.

Dada una curva $\alpha: I \rightarrow \mathbb{R}^{2}$ parametrizada regular, es decir, con $\alpha^{\prime}(t) \neq 0$ para toda $t \in I$, queremos asociarle una función $\kappa: I \rightarrow \mathbb{R}$ que en cada punto $\alpha(t)$ la cantidad $\kappa(t)$ indique "cuánto se curva la curva en dicho punto". La idea es encontrar la circunferencia que mejor aproxima a la curva $\alpha$ en el punto $P=\alpha\left(t_{0}\right)$. Tomamos dos puntos $Q=\alpha\left(t_{1}\right)$ y $R=\alpha\left(t_{2}\right)$ cercanos al punto $P=\alpha\left(t_{0}\right)$ y trazamos la circunferencia que pasa por $Q, P$ y $R$.

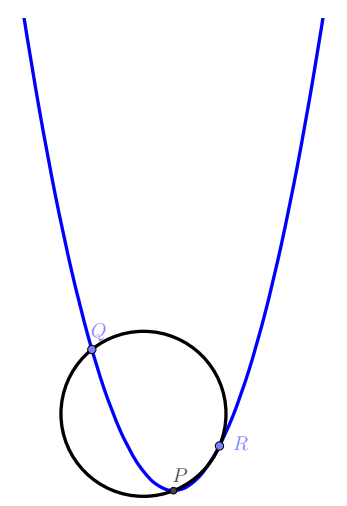

Hacemos tender $t_{1}$ y $t_{2}$ a $t_{0}$, es decir hacemos tender los puntos $Q$ y $R$ al punto $P$. 


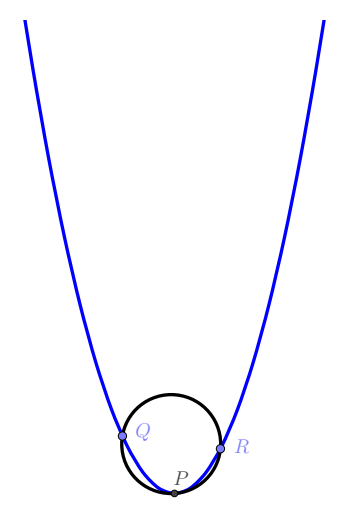

Si $\alpha^{\prime \prime}\left(t_{0}\right) \neq 0$, en el límite, obtenemos una circunferencia de radio $r_{P}$ la cual es llamada círculo osculador a $\alpha$ en $P$, para una demostración ver (Spivak, 1979, Chapter 1, Theorem 1). Entonces definimos la curvatura de la curva $\alpha$ en el punto $P=\alpha\left(t_{0}\right)$ por $\kappa\left(t_{0}\right)=\frac{1}{r_{P}}$. Si $\alpha^{\prime \prime}\left(t_{0}\right)=0$ definimos $\kappa\left(t_{0}\right)=0$.

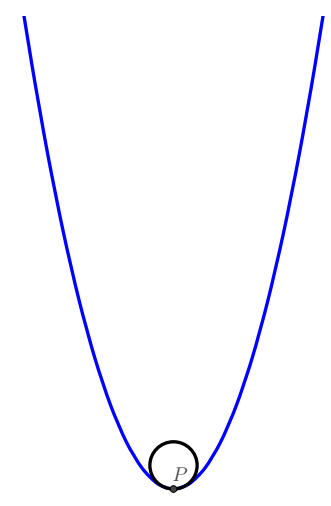

A esta noción de curvatura podemos asignar un signo que indica la dirección en que se desvía la curva. Asignamos el signo positivo cuando el centro del círculo osculador queda a la izquierda de la dirección de avance de la curva, y negativo en el caso contrario.

\section{Respuesta analítica}

Fijemos una recta en el plano, digamos el eje $x$. Dada una curva $\alpha: I \rightarrow \mathbb{R}^{2}$ regular parametrizada por longitud de arco, sea $\theta(t)$ el ángulo entre el vector tangente $\alpha^{\prime}(t)$ a la curva $\alpha$ en el punto $\alpha(t)$ y el eje $x$. 


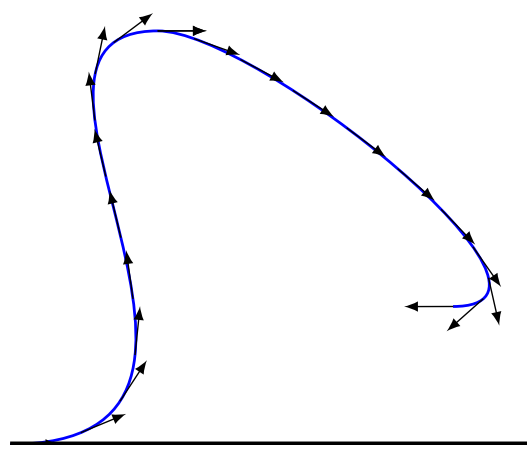

Intuitivamente, si la trayectoria es una recta, el ángulo no cambia y se mantiene constante. Por otro lado, si la trayectoria se curva mucho, el ángulo cambia muy rápido. Por lo tanto, definimos la curvatura como la razón de cambio del ángulo:

$$
\kappa(t)=\frac{d \theta}{d t}(t) .
$$

Esta definición no sólo nos da una magnitud, que mide la razón de la desviación de la curva de una recta, sino también un signo el cual indica la dirección de la desviación. El signo de la curvatura en un punto es:

Positivo si a partir de dicho punto la curva continúa hacia el lado izquierdo de la recta tangente que pasa por dicho punto, como se ilustra en la Figuras 3.

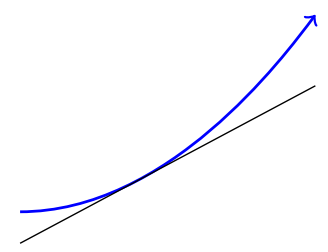

Figura 3: Curvatura positiva.

Negativo si a partir de dicho punto la curva continúa hacia el lado derecho de la recta tangente que pasa por dicho punto, como se muestra en la Figura 4

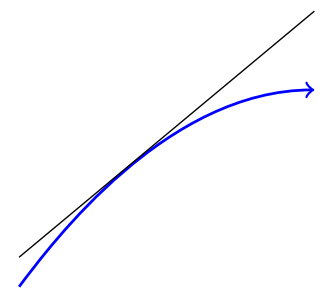

Figura 4: Curvatura negativa. 
Las dos nociones de curvatura coinciden si asignamos un signo a la noción geométrica como se indicó.

\section{Curvas en el espacio}

Ahora consideraremos curvas en el espacio Euclidiano $\mathbb{R}^{3}=\{(x, y, z) \mid x, y, z \in \mathbb{R}\}$ y definiremos su curvatura de manera analítica.

Sea $\alpha: I \rightarrow \mathbb{R}^{3}$ una curva diferenciable regular dada por $\alpha(t)=(x(t), y(t), z(t))$. Supongamos además que la curva $\alpha$ está parametrizada por longitud de arco, es decir, $\left\|\alpha^{\prime}(t)\right\|=1$ para toda $t \in I$. Como ejemplo, en la Figura 5 se muestra la curva en el espacio llamada hélice dada por $\alpha(t)=(a \cos t, a \sin t, b t)$, donde pedimos que $a^{2}+b^{2}=1$ para que esté parametrizada por longitud de arco.

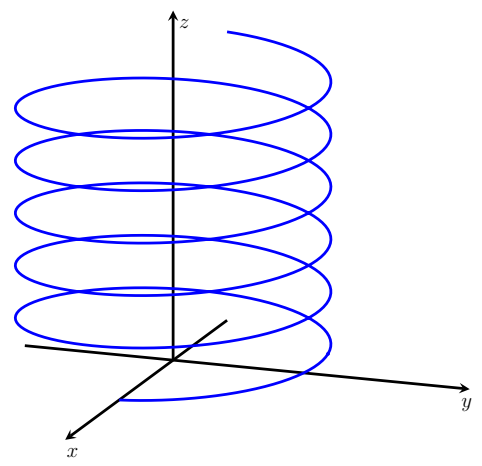

Figura 5: Curva en el espacio: Hélice.

Denotemos por $T(t)=\alpha^{\prime}(t)$ al vector tangente. Como $\|T(t)\|=\left\|\alpha^{\prime}(t)\right\|=1$ es constante, $T^{\prime}(t)$ mide la razón de cambio del ángulo que las tangentes cercanas hacen con la tangente en $t$. Por lo tanto definimos la curvatura de $\alpha$ en el punto $\alpha(t)$ por

$$
k(t)=\left\|T^{\prime}(t)\right\|=\left\|\alpha^{\prime \prime}(t)\right\| .
$$

Si la curva yace en un plano, las dos definiciones de curvatura coinciden salvo signo, es decir:

$$
k(t)=|\kappa(t)| .
$$

El vector $\alpha^{\prime \prime}(t)$ es ortogonal al vector tangente $\alpha^{\prime}(t)$, por esta razón, al vector unitario $n(t)$ en la dirección del vector $\alpha^{\prime \prime}(t)$ se le llama el vector normal a la curva $\alpha$ en el punto $\alpha(t)$. Además tenemos que $\alpha^{\prime \prime}(t)=k(t) n(t)$.

\section{Superficies}

El siguiente paso es estudiar superficies en el espacio Euclidiano $\mathbb{R}^{3}$. Así como en las secciones anteriores trabajamos con curvas parametrizadas regulares, que son curvas que en 
cada punto tienen un vector tangente no cero, ahora trabajaremos con superficies regulares, que son aquellas que tienen en cada punto un plano tangente bien definido.

Así como definimos una curva en $\mathbb{R}^{3}$ como una función de una variable real cuya imagen es un conjunto de $\mathbb{R}^{3}$, para definir una superficie es razonable considerar ahora funciones de dos variables reales $F: \mathbb{R}^{2} \rightarrow \mathbb{R}^{3}$. Entonces $F$ se puede escribir como una terna de funciones coordenadas, es decir

$$
F(u, v)=(x(u, v), y(u, v), z(u, v)) .
$$

Como pedimos que $F$ sea diferenciable, todas y cada una de sus funciones coordenadas son diferenciables, es decir, las derivadas parciales de todos los órdenes existen. Entonces podemos definir la derivada parcial $\frac{\partial F}{\partial u}$ de $F$ con respecto a $u$, también denotada por $F_{u}$, por

$$
\frac{\partial F}{\partial u}(u, v)=F_{u}(u, v)=\left(\frac{\partial x}{\partial u}(u, v), \frac{\partial y}{\partial u}(u, v), \frac{\partial z}{\partial u}(u, v)\right) .
$$

Análogamente podemos definir las restantes derivadas parciales de $F: \frac{\partial F}{\partial v}=F_{v}, \frac{\partial^{2} F}{\partial u^{2}}=F_{u u}$, $\frac{\partial^{2} F}{\partial u \partial v}=F_{u v}$, etc.

Una superficie parametrizada diferenciable regular en $\mathbb{R}^{3}$ es una función diferenciable $F: U \rightarrow \mathbb{R}^{3}$, con $U$ un conjunto abierto de $\mathbb{R}^{2}$, tal que los dos vectores dados por las derivadas parciales $F_{u}(u, v)$ y $F_{v}(u, v)$ son linealmente independientes para todo punto $(u, v)$ en $U$, o equivalentemente, que la matriz Jacobiana $D F_{q}$ de $F$, dada por

$$
D F_{q}=\left(\begin{array}{ll}
\frac{\partial x}{\partial u}(q) & \frac{\partial x}{\partial v}(q) \\
\frac{\partial y}{\partial u}(q) & \frac{\partial y}{\partial v}(q) \\
\frac{\partial z}{\partial u}(q) & \frac{\partial z}{\partial v}(q)
\end{array}\right)=\left(\begin{array}{ll}
F_{u}(q) & \left.F_{v}(q)\right)
\end{array}\right)
$$

tenga rango 2 para toda $q=(u, v)$ en $U$. A su vez, esto es equivalente a que el producto cruz $F_{u}(u, v) \times F_{v}(u, v)$ sea distinto de cero, es decir, que haya un vector normal bien definido en el punto $p=F(u, v)$. La traza de $F$ es el conjunto imagen $S=F(U)$. El plano tangente $T_{p} S$ a la superficie $F$ en el punto $p=F(u, v)$ es el plano generado por los vectores $F_{u}(u, v)$ y $F_{v}(u, v)$.

En general, si $A$ es un subconjunto arbitrario de $\mathbb{R}^{2}$, se dice que una aplicación $F: A \rightarrow \mathbb{R}^{3}$ es una superficie parametrizada, si $F$ puede ser extendida a una aplicación diferenciable de $U$ en $\mathbb{R}^{3}$, siendo $U$ un subconjunto abierto de $\mathbb{R}^{2}$ que contiene a $A$.

Ejemplo 1 (Coordenadas esféricas en la esfera.). Usando las coordenadas esféricas podemos ver a la esfera de radio $a>0$ como una superficie parametrizada regular dada por la aplicación $F:[0,2 \pi] \times(-\pi / 2, \pi / 2) \rightarrow \mathbb{R}^{3}$ definida por

$$
F(\phi, \theta)=(a \cos \theta \cos \phi, a \cos \theta \sin \phi, a \sin \theta) .
$$

Las lineas $\phi=$ constante son llamadas meridianos y las lineas $\theta=$ constante son llamadas paralelos o círculos de latitud. El círculo $\theta=0$ tambien es llamado ecuador; este es el único círculo máximo, de entre todos los paralelos. Todos los meridianos son círculos máximos. 


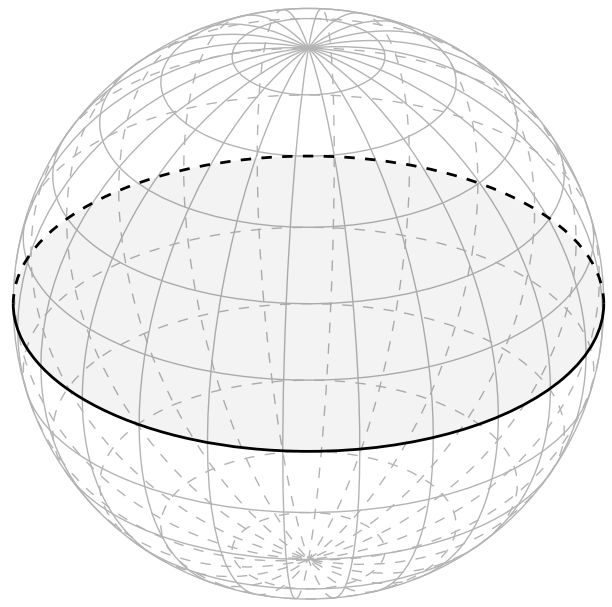

Figura 6: Coordenadas esféricas en la esfera.

Observaciones 1. La superficie parametrizada $F$ del Ejemplo 1 está definida sobre el rectángulo cerrado $[0,2 \pi] \times(-\pi / 2, \pi / 2)$, la cual admite una extensión diferenciable a un conjunto abierto de $\mathbb{R}^{2}$ que contiene a $[0,2 \pi] \times(-\pi / 2, \pi / 2)$. Sin embargo, cualquier extensión recubrirá más de una vez partes substanciales de la esfera, en tanto que $F$ solamente cubre dos veces a una semicircunferencia que va desde el polo norte al polo sur.

Existen ciertos subconjuntos de $\mathbb{R}^{3}$ a los que quisiéramos denominar superficies, pero que no encajan dentro del contexto de las superficies parametrizadas regulares descritas anteriormente. Tales subconjuntos no pueden ser descritos como la imagen biyectiva de una sola superficie parametrizada regular definida sobre un conjunto abierto de $\mathbb{R}^{2}$; como vimos, la esfera es un ejemplo de esto.

Introduciremos el concepto de superficie regular; intuitivamente se combinan convenientemente varias superficies parametrizadas para describir a ciertos subconjuntos de $\mathbb{R}^{3}$ que son los que queremos definir como superficies. La definición de superficie regular es un poco más complicada, pero es la que se generaliza a dimensiones superiores para dar el concepto de variedad diferenciable.

Definición 1. Un subconjunto $S \subset \mathbb{R}^{3}$ es una superficie regular si para cada $p \in S$, existe una vecindad $V$ en $\mathbb{R}^{3}$ y una aplicación $F: U \rightarrow V \cap S$ de un subconjunto abierto $U$ de $\mathbb{R}^{2}$ sobre $V \cap S \subset \mathbb{R}^{3}$, llamada parametrización de $S$, tal que

1. La aplicación $F$ es diferenciable.

2. La aplicación $F$ es un homeomorfismo: $F$ es continua (por la condición 1) y admite una inversa $F^{-1}: V \cap S \rightarrow U$ que es continua.

3. Cada aplicación $F: U \rightarrow S$ es una superficie parametrizada regular. 
Ejemplo 2. La esfera unitaria $\mathbb{S}^{2}=\left\{(x, y, z) \in \mathbb{R}^{3} \mid x^{2}+y^{2}+z^{2}=1\right\}$, es una superficie regular. Podemos cubrir a la esfera mediante seis superficies parametrizadas regulares cuyas trazas son hemisferios: $F_{i}: U \subset \mathbb{R}^{2} \rightarrow \mathbb{R}^{3}$ con $i=1, \ldots, 6$, dadas por

$$
\begin{array}{lll}
F_{1}(x, y)=\left(x, y,+\sqrt{1-\left(x^{2}+y^{2}\right)}\right), & (x, y) \in U, & U=\left\{(x, y) \in \mathbb{R}^{2} \mid x^{2}+y^{2}<1\right\}, \\
F_{2}(x, y)=\left(x, y,-\sqrt{1-\left(x^{2}+y^{2}\right)}\right), & (x, y) \in U, & U=\left\{(x, y) \in \mathbb{R}^{2} \mid x^{2}+y^{2}<1\right\}, \\
F_{3}(x, z)=\left(x,+\sqrt{1-\left(x^{2}+z^{2}\right)}, z\right), & (x, z) \in U, & U=\left\{(x, z) \in \mathbb{R}^{2} \mid x^{2}+z^{2}<1\right\}, \\
F_{4}(x, z)=\left(x,-\sqrt{1-\left(x^{2}+z^{2}\right)}, z\right), & (x, z) \in U, & U=\left\{(x, z) \in \mathbb{R}^{2} \mid x^{2}+z^{2}<1\right\}, \\
F_{5}(y, z)=\left(+\sqrt{1-\left(y^{2}+z^{2}\right)}, y, z\right), & (y, z) \in U, & U=\left\{(y, z) \in \mathbb{R}^{2} \mid y^{2}+z^{2}<1\right\}, \\
F_{6}(y, z)=\left(-\sqrt{1-\left(y^{2}+z^{2}\right)}, y, z\right), & (y, z) \in U, & U=\left\{(y, z) \in \mathbb{R}^{2} \mid y^{2}+z^{2}<1\right\} .
\end{array}
$$

Hay otra manera más práctica de obtener superficies regulares en $\mathbb{R}^{3}$, para ello necesitamos algunas definiciones.

Definición 2. Sea $f: \mathbb{R}^{3} \rightarrow \mathbb{R}$ una función diferenciable. El gradiente de $f$ en el punto $(x, y, z)$, denotado por $\nabla f(x, y, z)$ se define como

$$
\nabla f(x, y, z)=\left(\frac{\partial f}{\partial x}(x, y, z), \frac{\partial f}{\partial y}(x, y, z), \frac{\partial f}{\partial z}(x, y, z)\right) .
$$

Un punto $(x, y, z) \in \mathbb{R}^{3}$ es un punto crítico de $f$ si $\nabla f(x, y, z)=0$. La imagen $f(x, y, z) \in \mathbb{R}$ de un punto crítico es llamado un valor crítico de $f$. Un punto $a \in \mathbb{R}$ que no es un valor crítico es llamado un valor regular de $f$.

Teorema 1. Si $f: \mathbb{R}^{3} \rightarrow \mathbb{R}$ es una función diferenciable y $a \in \mathbb{R}$ es un valor regular de $f$, entonces $f^{-1}(a)$ es una superficie regular en $\mathbb{R}^{3}$.

Para una demostración ver (do Carmo, 1994, §2.2, Proposition 2).

Ejemplo 3. Consideremos la función $f: \mathbb{R}^{3} \rightarrow \mathbb{R}$ dada por $f(x, y, z)=x^{2}+y^{2}+z^{2}$. El gradiente está dado por $\nabla f(x, y, z)=(2 x, 2 y, 2 z)$. Es fácil ver que $1 \in \mathbb{R}$ es un valor regular de $f$ y por el Teorema 1 el conjunto

$$
f^{-1}(1)=\left\{(x, y, z) \in \mathbb{R}^{3} \mid f(x, y, z)=x^{2}+y^{2}+z^{2}=1\right\}
$$

es una superficie regular que es precisamente la esfera unitaria $\mathbb{S}^{2}$.

De ahora en adelante, en vez de decir superficie regular, solamente diremos superficie.

Usando el hecho de que sabemos calcular curvatura de curvas planas, definiremos las curvaturas normales en un punto de una superficie en $\mathbb{R}^{3}$. Entre las curvaturas normales definiremos las curvaturas principales, y con ellas, otros dos tipos de curvaturas: la curvatura Gaussiana y la curvatura media. 


\subsection{Curvatura normal}

Describiremos el proceso para definir las curvaturas normales de una superficie arbitraria $S$ y lo iremos ilustrando en el ejemplo de la superficie conocida como paraboloide hiperbólico o silla de montar, la cual está dada por

$$
\left\{(x, y, z) \in \mathbb{R}^{3} \mid z-x^{2}+y^{2}=0\right\} .
$$

Sea $S$ una superficie y sea $p \in S$.

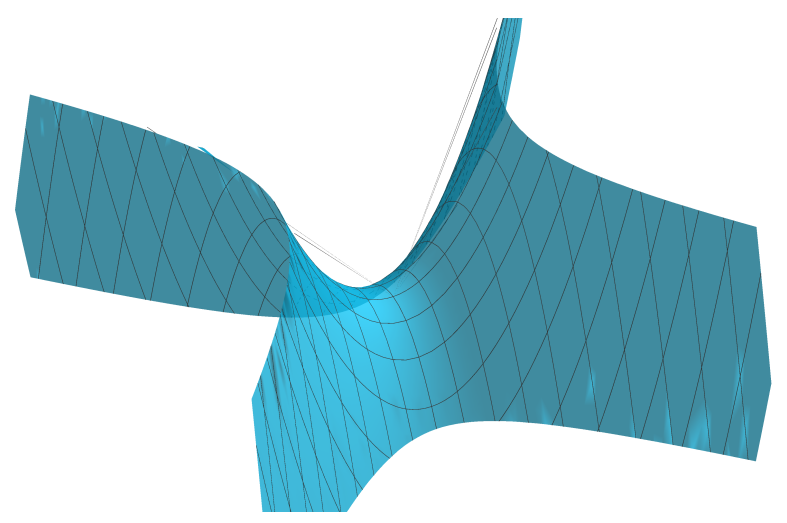

Tomemos el plano tangente $T_{p} S$ a $S$ en $p$.

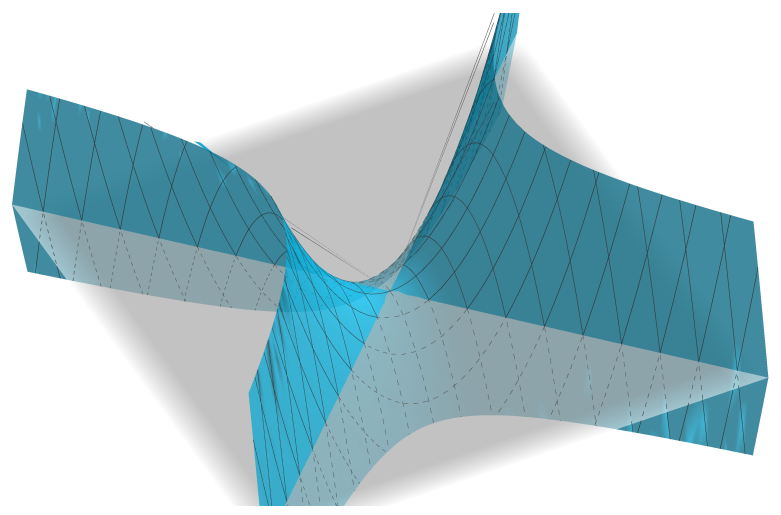

Tomemos un vector unitario normal $N$ a $T_{p} S$.

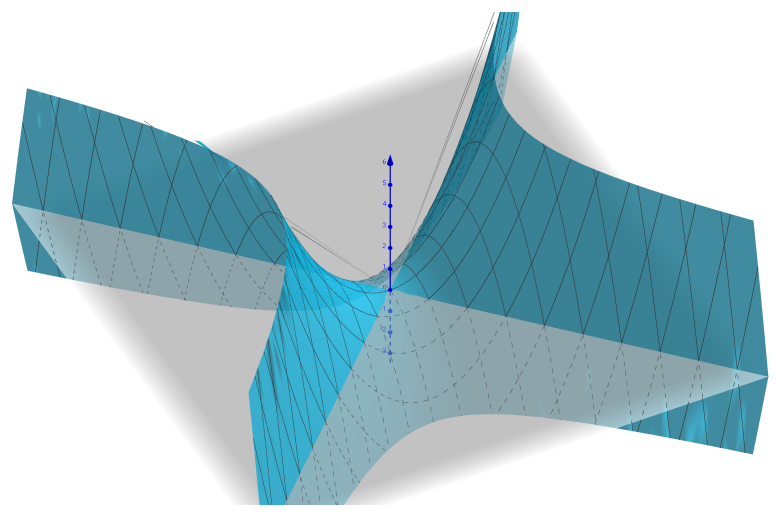


Sea $v$ en $T_{p} S$ y $P_{v}$ el plano normal a $S$ generado por $N$ y $v$.

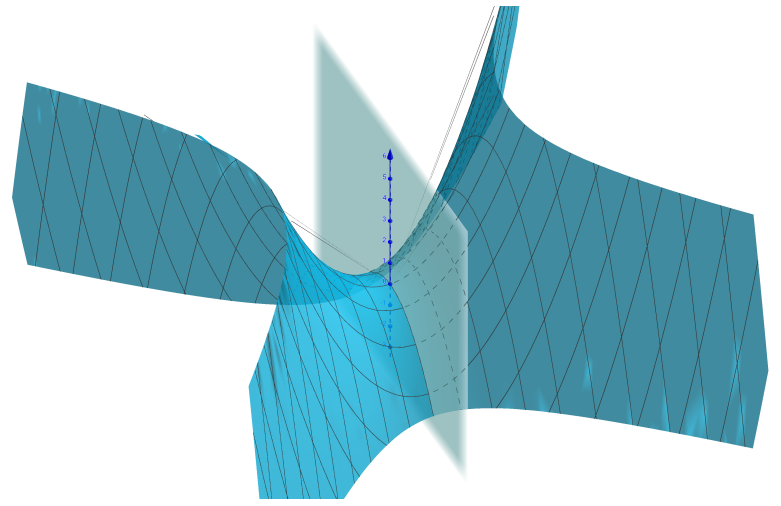

La intersección $P_{v} \cap S$ es una curva plana $\alpha_{v}$ contenida en $S$.

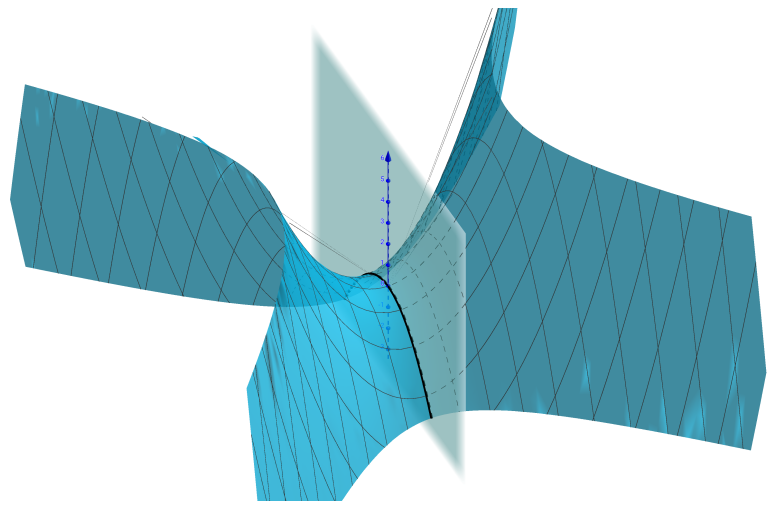

La curvatura normal de $S$ en $p$ en la dirección de $v$ es

$$
\kappa_{v}=\text { curvatura de } \alpha_{v} \text { en el tiempo } t_{0} \text {, tal que } \alpha\left(t_{0}\right)=p \text {. }
$$

Con signo positivo $(+)$ si $\alpha_{v}^{\prime \prime}\left(t_{0}\right)$ tiene el mismo sentido que $N$, es decir, $\left\langle\alpha^{\prime \prime}\left(t_{0}\right), N\right\rangle>0$, y signo negativo $(-)$ si $\alpha_{v}^{\prime \prime}\left(t_{0}\right)$ tiene el sentido opuesto que $N$, es decir, $\left\langle\alpha^{\prime \prime}\left(t_{0}\right), N\right\rangle<0$.

Si tomamos vectores tangentes $v$ en $T_{p} S$ distintos obtenemos curvaturas normales distintas como se muestra en la Figura 7 ;
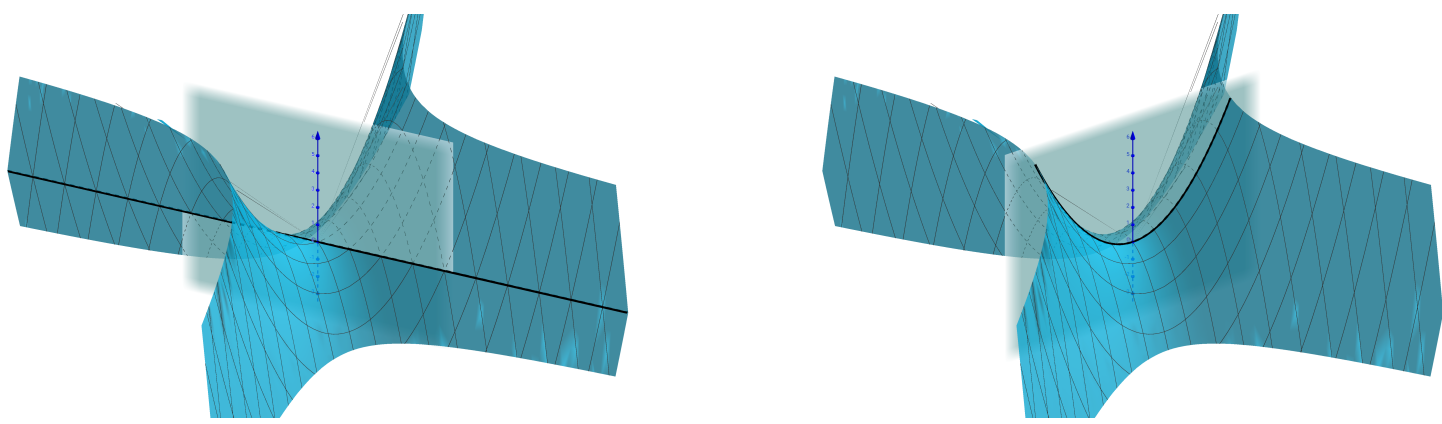

Figura 7: Otras dos curvaturas normales de $S$ en el punto $p$. 


\subsection{Curvatura normal y curvatura geodésica}

En la literatura a veces se da una definición alternativa de la curvatura normal, por ejemplo en (do Carmo, 1994, §3.2, Definición 3). Sea $S$ una superficie, $p \in S$ y $N$ un vector unitario normal a $S$ en $p$. Sea $C$ una curva regular en $S$ que pasa por $p$, parametrizada por $\alpha(s)$, donde $s$ es la longitud de arco de $C$ y $\alpha(0)=p$. Sea $n$ el vector normal a $C$ en $p$ y $\theta$ el ángulo entre los vectores $N$ y $n$. Sea $k=\left\|\alpha^{\prime \prime}(0)\right\|$ la curvatura de $C$ en $p$. El número $\kappa_{C}=k \cos \theta$ se denomina la curvatura normal de $C \subset S$ en $p$. Es decir, $\kappa_{C}$ es la longitud de la proyección $u$ del vector $k n=\alpha^{\prime \prime}(0)$ sobre la recta determinada por el vector normal $N$, esto se muestra en la Figura 8 .

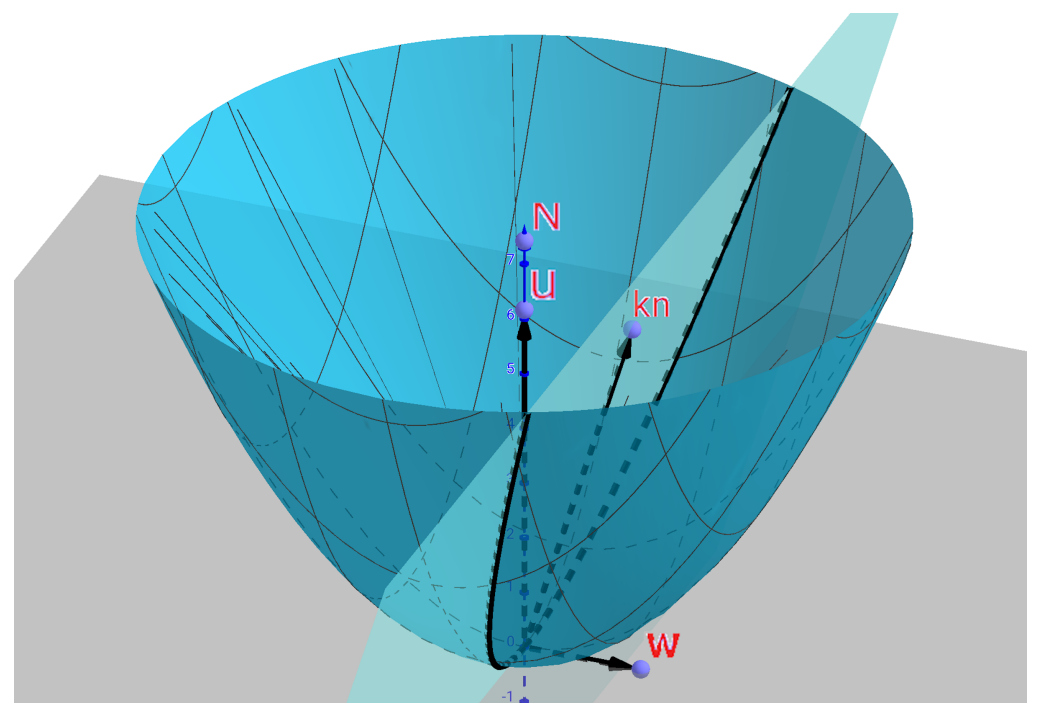

Figura 8: Curvaturas normal y geodésica.

Si $C$ es una curva que pasa por $p$ obtenida por la intersección de $S$ con un plano normal, tenemos que $\theta$ es cero y la curvatura normal de $C$ coincide con la curvatura $k$ de $C$, como en nuestra primera definición de curvatura normal. De hecho, se puede demostrar que todas las curvas contenidas en $S$ que tienen en un punto dado $p \in S$ la misma recta tangente, tienen en ese punto la misma curvatura normal (do Carmo, 1994, §3.2, Proposición 2). Por lo tanto, las dos definiciones de curvatura normal son equivalentes.

Por otro lado, consideremos la proyección $w$ del vector $k n=\alpha^{\prime \prime}(0)$ sobre el espacio tangente $T_{p} S$ (ver Figura 8), a la longitud de $w$ se le denota por $\kappa_{g}$ y se le llama la curvatura geodésica de $C$ en $p$, es decir, $k_{g}=k \operatorname{sen} \theta$. Por lo tanto tenemos que $k^{2}=\kappa_{C}^{2}+\kappa_{g}^{2}$.

\subsection{Curvaturas principales}

A cada vector unitario $v \in T_{p} S$ le asociamos la curvatura normal $\kappa_{v}$ de $S$ en $p$ en la dirección de $v$. Esto nos da una función continua (en la sección 5.5 se explica porqué es 
continua)

$$
\begin{aligned}
k: \mathbb{S}^{1} & \rightarrow \mathbb{R} \\
v & \mapsto \kappa_{v},
\end{aligned}
$$

donde $\mathbb{S}^{1}$ es la circunferencia unitaria en $T_{p} S$. El máximo $\kappa_{1}$ y el mínimo $\kappa_{2}$ de las curvaturas normales son llamadas las curvaturas principales de $S$ en $p$. Los vectores unitarios $e_{1}$ y $e_{2}$ en cuyas direcciones se alcanzan $\kappa_{1}$ y $\kappa_{2}$ son llamados las direcciones principales en $p$.

En el ejemplo de la silla de montar las curvaturas principales se muestran en la Figura 9 ;

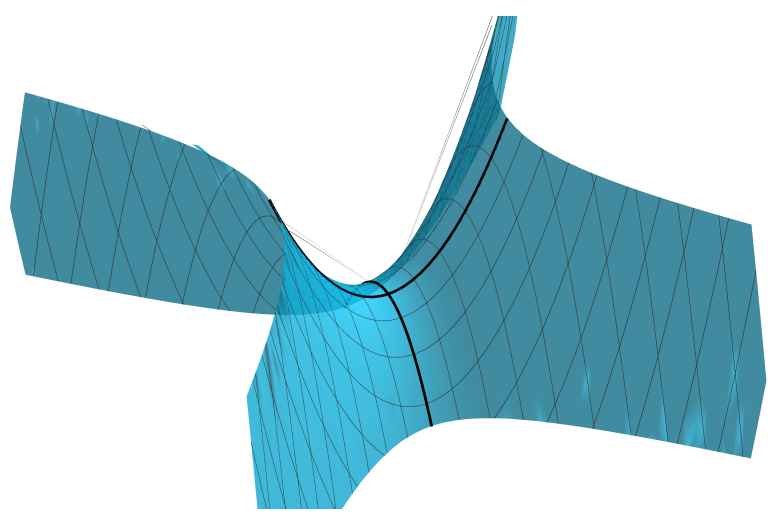

Figura 9: Curvaturas principales.

\subsection{Curvatura media y curvatura Gaussiana}

A partir de las curvaturas principales definimos:

Curvatura Gaussiana: La curvatura Gaussiana $K(p)$ de una superficie $S$ en un punto $p \in S$ es el producto de las curvaturas principales de $S$ en $p$ :

$$
K=\kappa_{1} \kappa_{2}
$$

Curvatura media La curvatura media $H(p)$ de una superficie $S$ en un punto $p \in S$ es el promedio de las curvaturas principales de $S$ en $p$ :

$$
H=\frac{\kappa_{1}+\kappa_{2}}{2} .
$$

La curvatura Gaussiana define diferentes tipos de puntos en una superficie.

Punto silla o punto hiperbólico. Un punto $p$ en una superficie $S$ es un punto hiperbólico si $K(p)<0$. Esto es equivalente a que las curvaturas principales $\kappa_{1}$ y $\kappa_{2}$ en $p$ tienen signos opuestos, y a su vez esto implica que $S$ cruza el plano tangente en cualquier vecindad de $p$. 


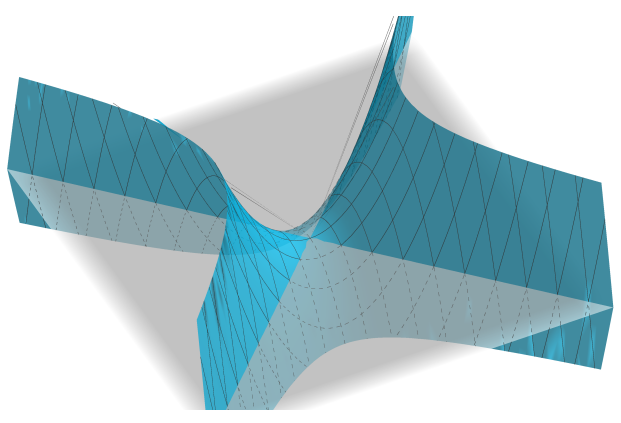

Figura 10: Punto hiperbólico.

Punto plano o punto parabólico. Un punto $p$ en una superficie $S$ es un punto parabólico si $K(p)=0$. Esto es equivalente a que alguna curvatura principal $\kappa_{1}, \kappa_{2}$ (o ambas) sea cero.

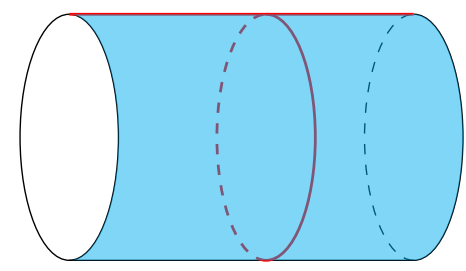

Figura 11: Punto parabólico.

Punto elíptico. Un punto $p$ en una superficie $S$ es un punto elíptico si $K(p)>0$. Esto es equivalente a que las curvaturas principales $\kappa_{1}$ y $\kappa_{2}$ tengan el mismo signo, y a su vez esto implica que $S$ está en un lado del plano tangente a $p$, del mismo lado que $n$ si $\kappa_{1}, \kappa_{2}>0$, del lado opuesto que $n$ si $\kappa_{1}, \kappa_{2}<0$.

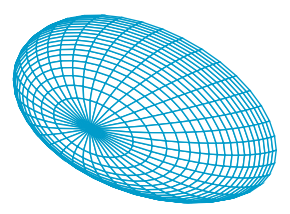

Figura 12: Punto elíptico.

Hay superficies que tienen los tres tipos de puntos. Por ejemplo el toro ${ }^{\mathbb{1}}$ dado por la parametrización $F:[0,2 \pi] \times[0,2 \pi]$

$$
F(u, v)=((2+\cos u) \cos v,(2+\cos u) \sin v, \sin u) .
$$




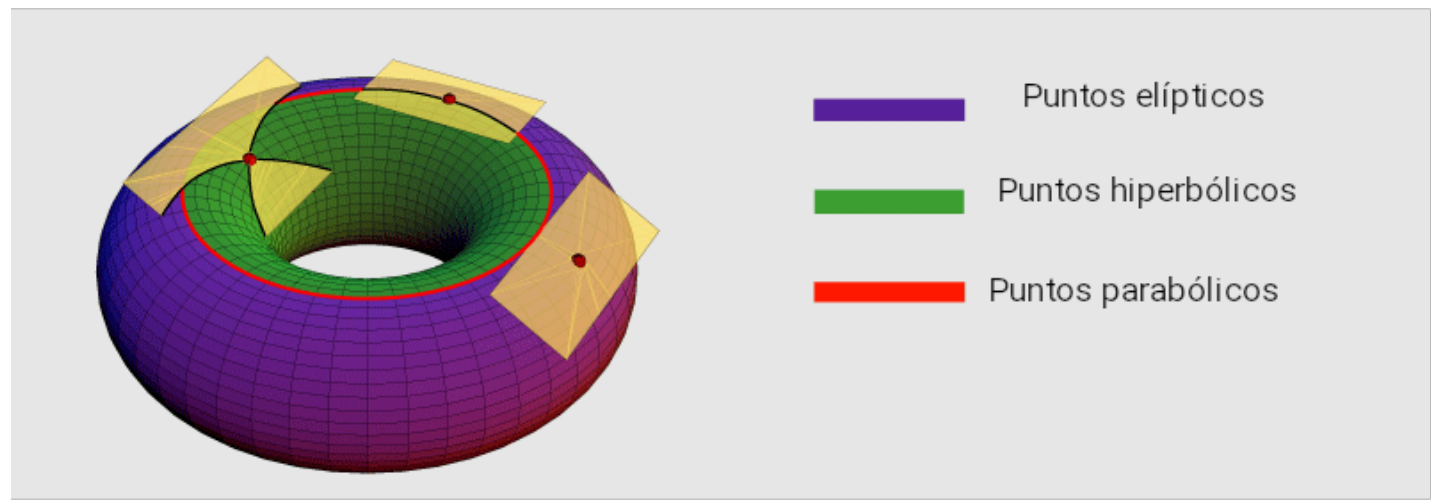

\subsection{Las aplicaciones de Gauss y Weingarten}

Ahora presentaremos una manera analítica de obtener la curvatura Gaussiana y la curvatura media.

Sea $S$ una superficie en $\mathbb{R}^{3}$. Sea $p \in S$ y $V$ una vecindad abierta de $p$ en $S$. La aplicación de Gauss $N: V \rightarrow \mathbb{S}^{2}$ es una aplicación diferenciable que asocia a cada punto $p \in V \subset S$ un vector normal unitario como se ilustra en la Figura 13
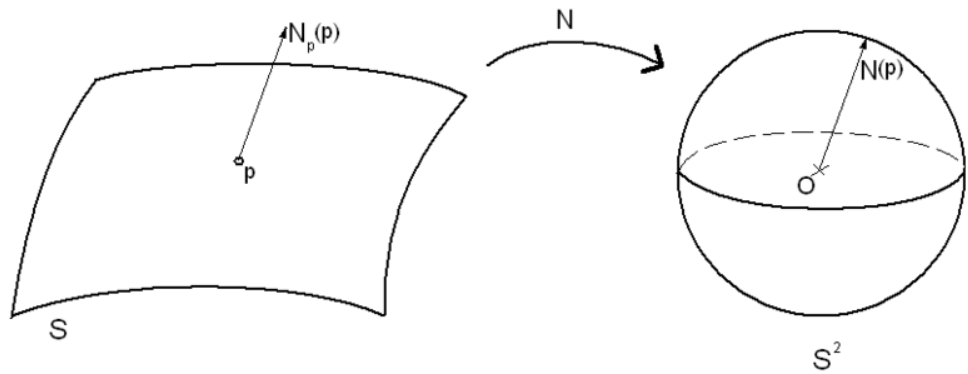

Figura 13: Aplicación de Gauss.

Una manera de dar la aplicación de Gauss es la siguiente. Sea $F: U \rightarrow S \subset \mathbb{R}^{3}$ una parametrización de $S$, con $U$ un abierto de $\mathbb{R}^{2}$ y la traza de $F$ es $V=F(U)$. Sean $q \in U$ y $p=F(q)$, entonces la aplicación de Gauss está dada por el producto cruz en $\mathbb{R}^{3}$ de las derivadas parciales de $F$ normalizado:

$$
N(p)=\frac{F_{u}(q) \times F_{v}(q)}{\left\|F_{u}(q) \times F_{v}(q)\right\|} .
$$

La diferencial de la aplicación de Gauss, conocida como aplicación de Weingarten, es una aplicación lineal $d N_{p}: T_{p} S \rightarrow T_{N(p)} \mathbb{S}^{2}$. Como $T_{p} S$ y $T_{N(p)} \mathbb{S}^{2}$ son planos paralelos, $d N_{p}$ puede verse como una aplicación lineal de $T_{p} S$ en sí mismo $d N_{p}: T_{p} S \rightarrow T_{p} S$. Una propiedad muy importante de la aplicación de Weingarten es que es autoadjunta, es decir, satisface la siguiente igualdad (do Carmo, 1994 , Sección 3.2, Proposición 1)

$$
\left\langle d N_{p}\left(v_{1}\right), v_{2}\right\rangle=\left\langle v_{1}, d N_{p}\left(v_{2}\right)\right\rangle, \quad \text { para todo } v_{1}, v_{2} \in T_{p} S,
$$


donde $\langle$,$\rangle es el producto interior en \mathbb{R}^{3}$ restringido a $T_{p} S$. Esto implica (ver do Carmo, 1994. Teorema, Apéndice del Capítulo 3)) que existe una base ortonormal $\left\{e_{1}, e_{2}\right\}$ de $T_{p} S$, que corresponde a las direcciones principales, tal que

$$
d N_{p}\left(e_{i}\right)=-\kappa_{i} e_{i}, \quad \text { con } i=1,2,
$$

es decir, las direcciones principales son vectores propios de $d N_{p}$ con valor propio $-\kappa_{i}$. Por lo tanto, $e_{1}$ y $e_{2}$ forman una base ortonormal que diagonaliza a $d N_{p}$. Entonces $d N_{p}=\left(\begin{array}{cc}-\kappa_{1} & 0 \\ 0 & -\kappa_{2}\end{array}\right)$ y

$$
K=\operatorname{det} d N_{p}, \quad H=-\frac{1}{2} \operatorname{traza} d N_{p} .
$$

Podemos definir una forma cuadrática $Q: T_{p} S \rightarrow \mathbb{R}$ mediante $Q(v)=-\left\langle d N_{p}(v), v\right\rangle$, la cual restringida a la circunferencia unitaria en $T_{p} S$ da la función $k: \mathbb{S}^{1} \rightarrow \mathbb{R}$ definida en (1), es decir, $k(v)=\left.Q\right|_{\mathbb{S}^{1}}(v)=\kappa_{v}$, y por esta razón la función $k$ es continua.

\subsection{Superficies orientables y no orientables}

Como vimos en la Subsección 5.5, dada una parametrización $F: U \rightarrow S \subset \mathbb{R}^{3}$ de una superficie $S$, con $U$ un abierto de $\mathbb{R}^{2}$, la aplicación de Gauss asocia a cada punto $p=F(q)$ en la imagen de $F$ el vector normal dado por el producto cruz de las derivadas parciales de $F$, es decir, $N(p)=\frac{F_{u}(q) \times F_{v}(q)}{\left\|F_{u}(q) \times F_{v}(q)\right\|}$ para toda $q \in V$. En otras palabras, la aplicación de Gauss define un campo vectorial normal diferenciable sobre el subconjunto abierto $V=F(U)$ de $S$. Un hecho sorprendente es que no todas las superficies admiten un campo vectorial normal diferenciable definido globalmente sobre toda la superficie. Un ejemplo de dichas superficies son la banda de Möbius ${ }^{3}$ y la botella de Klein ${ }^{\mathbb{4}}$ mostradas en la Figura 14. Diremos que dichas superficies son no orientables.

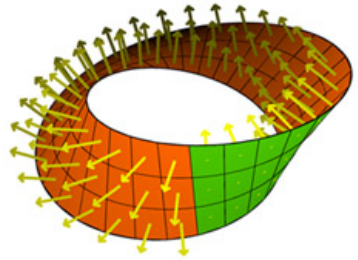

(a) Banda de Möbius

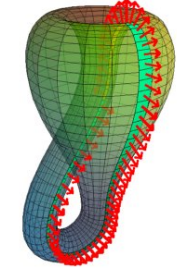

(b) Botella de Klein

Figura 14: Superficies no orientables.

Por otro lado, diremos que una superficie $S$ es orientable si admite un campo vectorial normal diferenciable $N$ definido en toda la superficie. La elección de dicho campo $N$ es llamada una orientación de $S$. Ejemplos de superficies orientables son la esfer $a^{5}$ y el tor $\sigma^{6}$ mostrados en la Figura 15. 


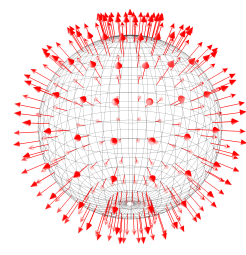

(a) Esfera

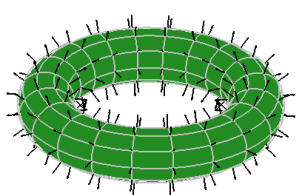

1

(b) Toro

Figura 15: Superficies orientables.

Por lo tanto, si $S$ es una superficie orientable, escoger una orientación es equivalente a dar una aplicación de Gauss global, es decir, definida sobre toda la superficie $N: S \rightarrow \mathbb{S}^{2} \subset \mathbb{R}^{3}$. En este caso, la curvaturas de Gauss $K: S \rightarrow \mathbb{R}$ y media $H: S \rightarrow \mathbb{R}$ también están bien definidas sobre toda la superficie.

\section{Dimensiones superiores: variedades Riemannianas}

Como mencionamos en la Sección 5, el concepto de superficie regular se puede generalizar a dimensiones superiores para obtener subconjuntos de $\mathbb{R}^{n}$ tales que en cada punto tengan bien definido un espacio tangente, el cual es un subespacio de $\mathbb{R}^{n}$ de dimensión $k \leq n$.

Definición 3. Un subconjunto $M \subset \mathbb{R}^{n}$ es una variedad diferenciable de dimensión $0<k \leq$ $n$ si para cada $p \in M$, existe una vecindad $V$ en $\mathbb{R}^{n}$ y una aplicación $F: U \rightarrow V \cap M$ de un subconjunto abierto $U$ de $\mathbb{R}^{k}$ sobre $V \cap M \subset \mathbb{R}^{n}$ de la forma

$$
F\left(u_{1}, \ldots, u_{k}\right)=\left(x_{1}\left(u_{1}, \ldots, u_{k}\right), \ldots, x_{n}\left(u_{1}, \ldots, u_{k}\right)\right)
$$

tal que

1. La aplicación $F$ es diferenciable.

2. La aplicación $F$ es un homeomorfismo.

3. Cada aplicación $F: U \rightarrow M$ tiene matriz Jacobiana

$$
D F_{q}=\left(\begin{array}{ccc}
\frac{\partial x_{1}}{\partial u_{1}}(q) & \ldots & \frac{\partial x_{1}}{\partial u_{k}}(q) \\
\vdots & \ddots & \vdots \\
\frac{\partial x_{n}}{\partial u_{1}}(q) & \ldots & \frac{\partial x_{n}}{\partial u_{k}}(q)
\end{array}\right)=\left(\begin{array}{lll}
F_{u_{1}}(q) & \ldots & \left.F_{u_{k}}(q)\right)
\end{array}\right)
$$

de rango $k$ para todo punto $q=\left(u_{1}, \ldots, u_{k}\right) \in U \subset \mathbb{R}^{k}$. 
Como en el caso de las superficies regulares, la condición 3 es equivalente a que los $k$ vectores en $\mathbb{R}^{n}$ dados por las derivadas parciales $F_{u_{i}}(q)$ con $i=1, \ldots, k$, sean linealmente independientes. El espacio tangente $T_{p} M$ a la variedad $M$ en el punto $p=F(q)$ es el subespacio generado por los vectores $F_{u_{1}}(q), \ldots, F_{u_{k}}(q)$. Como $T_{p} M$ es un subespacio de $\mathbb{R}^{n}$, podemos dotar a $T_{p} M$ de un producto interior restringiendo el producto interior estandar de $\mathbb{R}^{n}$. Una variedad diferenciable $M$ dotada de un producto interior en cada espacio tangente $T_{p} M$ que varía diferenciablemente es llamada una variedad Riemanniana.

En el plano $\mathbb{R}^{2}$, dados dos puntos, la línea de menor longitud que los une es el segmento de recta que pasa por ambos puntos. Análogamente, dados dos puntos en el espacio Euclidiano de dimensión $n$ la línea de menor longitud que los une es una recta. En general, dada una variedad Riemanniana de dimensión $k$ en $\mathbb{R}^{n}$, existen curvas de menor longitud que unen dos puntos las cuales son llamadas geodésicas. En una superficie $S$, una curva $C$ es una geodésica si la curvatura geodésica $\kappa_{g}$ de $C$ en todo punto es cero. Por ejemplo, en la esfera $\mathbb{S}^{2}$ las geodésicas son los círculos máximos, como los meridianos o el ecuador en el Ejemplo 1. Ésto es fácil de ver, pues los círculos máximos se obtienen de intersectar a la esfera con planos que pasan por el centro de la esfera, dichos planos son planos normales a la esfera, por lo tanto, la curvatura geodésica de los circulos máximos es cero. Para saber más sobre geodésicas recomiendo el artículo (Simanca, 2002).

\section{Hipersuperficies en $\mathbb{R}^{n}$}

Una hipersuperficie en $\mathbb{R}^{n}$ es una variedad diferenciable de dimensión $n-1$ (codimensión 1). Sea $S \subset \mathbb{R}^{n}$ una hipersuperficie. Podemos definir curvaturas normales como en el caso de superficies en $\mathbb{R}^{3}$. Sean $p \in S$, un vector normal unitario $N$ en $p$ y un vector tangente unitario $v \in T_{p} S$. La intersección del plano generado por $N$ y $v$ y $S$ es una curva, cuya curvatura $\kappa_{v}$ es la curvatura normal de $S$ en $p$ en la dirección de $v$. La aplicación $v \mapsto \kappa_{v}$ es la restricción a la esfera unitaria de una forma cuadrática $Q$ en $T_{p} S$. Existe una base ortonormal $\left\{e_{1}, \ldots, e_{n-1}\right\}$ de $T_{p} S$ que diagonaliza a $Q$. Las direcciones $e_{1}, \ldots, e_{n-1}$ son llamadas las direcciones de curvatura principales en $p$ y las cantidades correspondientes

$$
\kappa_{1}=\kappa_{e_{1}}, \ldots, \kappa_{n}=\kappa_{e_{n-1}}
$$

son las curvaturas principales de $S$ en $p$.

Como en $\mathbb{R}^{3}$ podemos considerar la aplicación de Gauss

$$
N: S \rightarrow \mathbb{S}^{n-1}
$$

cuya diferencial

$$
d N_{p}: T_{p} S \rightarrow T_{N(p)} \mathbb{S}^{n-1} \cong T_{p} S
$$

satisface las ecuaciones

$$
d N_{p}\left(e_{i}\right)=-\kappa_{i} e_{i}, \quad i=1, \ldots, n-1 .
$$

Definimos la curvatura media

$$
H=\frac{1}{n-1}\left(\kappa_{1}+\cdots+\kappa_{n-1}\right)=-\frac{1}{n-1} \operatorname{traza}\left(d N_{p}\right),
$$


y la curvatura de Gauss-Kronecker

$$
\kappa_{1} \ldots \kappa_{n-1}=(-1)^{n-1} \operatorname{det}\left(d N_{p}\right) .
$$

Además, tenemos las otras funciones simétricas elementales de las curvaturas principales, llamadas curvaturas medias de orden superior. Denotemos por $H_{j}$ a la curvatura media de orden j-ésimo, normalizada tal que

$$
\prod_{j=1}^{n-1}\left(1+t \kappa_{j}\right)=\sum_{j=0}^{n-1}\left(\begin{array}{c}
n-1 \\
j
\end{array}\right) H_{j} t^{j} .
$$

Entonces $H_{1}=H$, la curvatura media y $H_{n-1}$ es la curvatura de Gauss-Kronecker.

En dimensiones superiores tenemos curvaturas intermedias. Entre ellas $H_{2}$ juega un papel importante. Salvo por una constante, $H_{2}$ es igual a la curvatura escalar, la cual definiremos en la siguiente sección.

\section{Curvatura seccional y curvatura escalar}

Sea $M$ una variedad Riemanniana de dimensión $k$ en $\mathbb{R}^{n}$. Riemann definió en cada punto $p \in M$ las curvaturas seccionales: sean $v, w \in T_{p} M$ linealmente independientes. Para todo vector unitario

$$
u=\lambda v+\mu w
$$

existe una única geodésica en $M$ que empieza en $p$ con vector tangente $u$ (ver (do Carmo, 1992, Ch. 3, Proposition 2.7)).

Dichas geodésicas, al variar $u$ generan una superficie cuya curvatura Gaussiana en $p$ es llamada la curvatura seccional $K_{\pi}=K[v, w]$ del plano $\pi$ generado por $v$ y $w$.

Sea $\beta=\left\{e_{1}, \ldots, e_{k}\right\}$ una base ortonormal de $T_{p} M$. Entonces la cantidad

$$
s=2 \sum_{1 \leq i<j \leq k} K\left[e_{i}, e_{j}\right]
$$

es independiente de $\beta$ (ver (do Carmo, 1992, §4.4)), llamada la curvatura escalar de $M$ en $p$.

Si $M$ es una hipersuperfice en $\mathbb{R}^{n}$ podemos considerar a $e_{i}$ como las direcciones principales en $p$. Entonces $K\left[e_{i}, e_{j}\right]=\kappa_{i} \kappa_{j}$ por lo que

$$
s=\sum_{1 \leq i<j \leq k} \kappa_{i} \kappa_{j}, \quad \text { o } \quad s=(n-1)(n-2) H_{2} .
$$

\section{Resultados clásicos sobre curvatura}

Ya que hemos visto diferentes tipos de curvaturas, en esta sección enunciaremos algunos resultados clásicos que las involucran. Para ver más resultados relacionados con curvaturas recomiendo el artículo panorámico de Osserman (Osserman, 1990) el cual en parte motivó al presente artículo. 


\subsection{Cantidades intrínsecas y extrínsecas}

Sea $M \subset \mathbb{R}^{n}$ una variedad Riemanniana. Las cantidades intrínsecas de $M$ son determinadas mediante mediciones sobre $M$ misma. Las cantidades extrínsecas dependen del encaje de $M$ en $\mathbb{R}^{n}$, es decir, de "cómo está metida" $M$ en $\mathbb{R}^{n}$. Para $S \subset \mathbb{R}^{3}$ las curvaturas principales y la curvatura media son extrínsecas: enrollar una hoja en un cilindro las cambia, pero no cambian las distancias a lo largo de $S$. Sorprendentemente, el Teorema Egregio de Gauss (do Carmo, 1994, §4-3) dice que la Curvatura Gaussiana $K=\kappa_{1} \kappa_{2}$ es intrínseca. Para una discusión sobre porpiedades intrínsecas vs. extrínsecas recomiendo el artículo (Palmas, 2020).

\subsection{Teorema de Gauss-Bonnet}

Antes de poder enunciar el Teorema de Gauss-Bonnet necesitamos dar algunas definiciones.

Una superficie es compacta si es compacta como espacio topológico (cerrado y acotado como subespacio de $\mathbb{R}^{3}$ ). Algunas superficies tienen frontera, como la banda de Möbius. Una superficie cerrada es una superficie compacta y sin frontera. Ejemplos de superficies cerradas son: la esfera, el toro y la botella de Klein.

Recordemos que dos espacios topológicos son homeomorfos si existe un homeomorfismo entre ellos, es decir, una aplicación biyectiva continua cuya inversa también es continua. En topología dos espacios homeomorfos son considerados equivalentes.

El Teorema de clasificación de superficies (ver por ejemplo (S. William Massey, 1967)) nos dice que toda superficie cerrada orientada es homeomorfa a una superficie de la siguiente lista: la esfera, el toro, el toro con dos hoyos, etc., como se muestra en la Figura 1 fop.

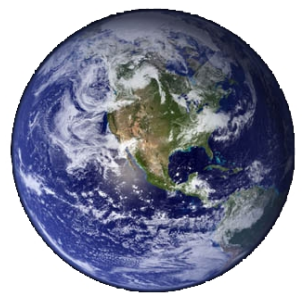

(a) Género 0

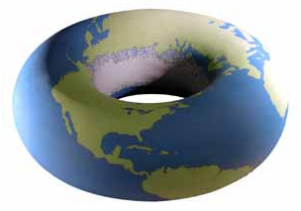

(b) Género 1

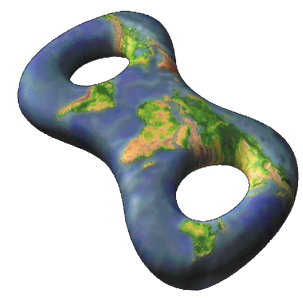

(c) Género 2

Figura 16: Superficies cerradas orientadas

Distinguimos las superficies de la lista anterior por que tienen diferente género, que intuitivamente corresponde al "número de hoyos".

A toda superficie cerrada orientada $S$ podemos asociarle un número entero $\chi(S)$ llamado la característica de Euler-Poincaré de $S$. Una manera de calcular a $\chi(S)$ es la siguiente. Un resultado muy importante en topología es que las superficies compactas se pueden triangular (ver (Doyle and Moran, 1968)), es decir, podemos encontrar un poliedro $P$ que sea homeomorfo a una superficie $S$ dada. En la Figura 17 se muestran triangulaciones de la esfera ${ }^{[8}$ y del toro 9 : 

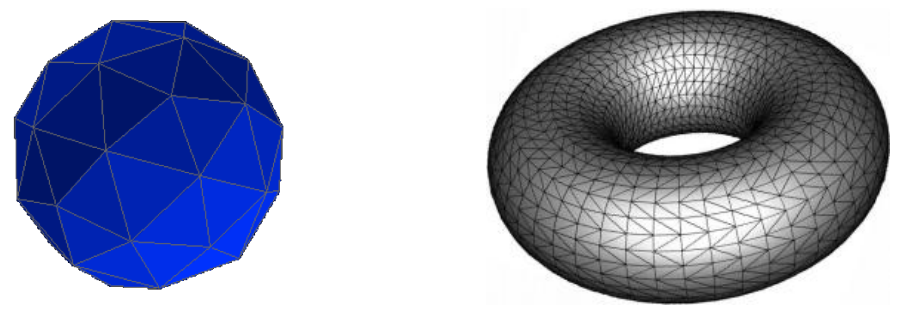

Figura 17: Triangulaciones de la esfera y el toro.

La característica de Euler-Poincaré de la superficie $S$ se define como $\chi(S)=V-A+C$, donde $V$ es el número de vértices, $A$ el de aristas y $C$ el de caras de una triangulación $P$, y no depende de la triangulación, por lo que es un invariante topológico, el cual es completo, es decir: dos superficies cerradas orientadas $S$ y $S^{\prime}$ son homeomorfas si y sólo si $\chi(S)=\chi\left(S^{\prime}\right)$. Por ejemplo, la esfera tiene característica de Euler-Poincaré igual a 2 (un tetraedro es una triangulación de la esfera y ahí es fácil calcularla), por lo tanto, toda superficie cerrada y orientada $S$ con $\chi(S)=2$ es homeomorfa a la esfera. Si denotamos por $T_{g}$ a la superficie de género $g$ (toro con $g$ hoyos) tenemos que $\chi\left(T_{g}\right)=2-2 g$, por lo tanto, la única superficie cerrada y orientada con característica de Euler-Poincaré positiva es la esfera.

Sea $S$ una superficie orientada. Una curva parametrizada regular a trozos cerrada y simple $\alpha: I \rightarrow S$ es una curva cerrada, sin autointersecciones, cuya recta tangente no está definida sólamente en un número finito de puntos $t_{0}, \ldots, t_{k} \in I$, los puntos $\alpha\left(t_{i}\right), i=1, \ldots, k$, son llamados los vértices de $\alpha$ y las trazas $\alpha\left(\left[t_{i}, t_{i+1}\right]\right)$ son los arcos regulares de $\alpha$. En cada vértice $\alpha\left(t_{i}\right)$ definimos el ángulo exterior $\theta_{i}$ como el ángulo formado por los límites izquierdo y derecho respectivamente cuando $t \rightarrow t_{i}$, de los vectores tangentes $\alpha^{\prime}(t)$ con $t \in\left[t_{i-1}, t_{i}\right]$ y $t \in\left[t_{i}, t_{i+1}\right]$ respectivamente. El signo del ángulo $\theta_{i}$ está dado por la orientación de $S$. El ángulo interior $\eta_{i}$ en el vértice $\alpha\left(t_{i}\right)$ está dado por $\eta_{i}=\pi-\theta_{i}$.

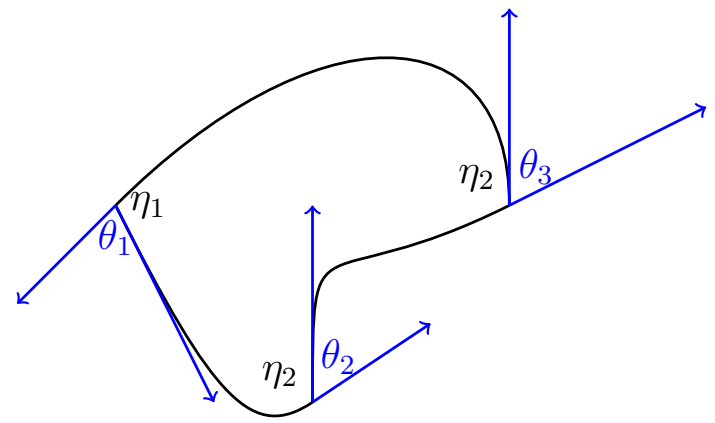

Figura 18: Ángulos exteriores e interiores de una curva parametrizada regular a trozos.

Sea $S$ una superficie orientada. Una subconjunto $R$ de $S$ es una región simple si $R$ es homeomorfa a un disco y la frontera $\partial R$ de $R$ es una curva parametrizada regular a trozos cerrada y simple $\alpha: I \rightarrow S$. Intuitivamente la frontera $\alpha$ está orientada positivamente si al caminar sobre la curva en la dirección positiva, con la cabeza apuntando en la dirección del 
vector normal $N$ que da la orientación a $S$, la región $R$ queda a la izquierda. Llamaremos triángulo a una región simple con sólo tres vértices como la mostrada en la Figura 19.

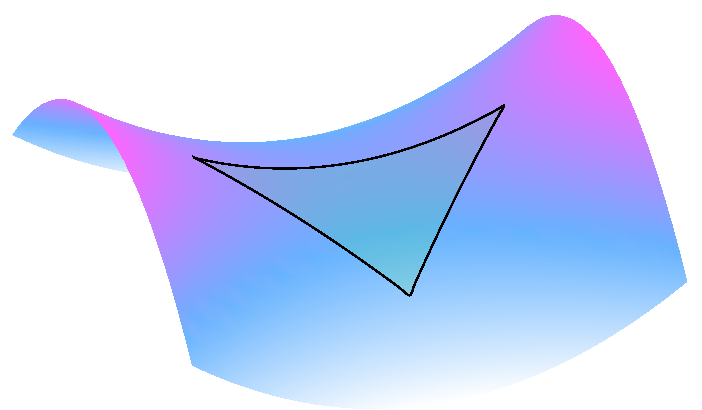

Figura 19: Región simple (triángulo) en una superficie.

Decimos que una región $R \subset S$ es regular si $R$ es compacta y su frontera $\partial R$ es la unión de un número finito de curvas regulares a trozos, cerradas y simples que no se cortan. Consideraremos a una superficie cerrada como una región regular sin frontera.

Ahora podemos enunciar el Teorema de Gauss-Bonnet.

Teorema 2 (Teorema de Gauss-Bonnet(do Carmo, 1994, §4.5)). Sea $R$ una región regular de una superficie orientada y sean $C_{1}, \ldots, C_{n}$ las curvas regulares a trozos cerradas y simples que forman la frontera $\partial R$ de $R$. Supongamos que cada $C_{i}$ está orientada positivamente $y$ sean $\theta_{1}, \ldots \theta_{p}$ el conjunto de todos los ángulos externos de las curvas $C_{1}, \ldots, C_{n}$. Entonces

$$
\sum_{i=1}^{n} \int_{C_{i}} \kappa_{g}(s) d s+\iint_{R} K \mathrm{dvol}+\sum_{l=1}^{p} \theta_{l}=2 \pi \chi(R) .
$$

donde $s$ denota la longitud de arco de $C_{i}$ y la integral sobre $C_{i}$ significa la suma de las integrales que corresponden a los arcos regulares de $C_{i}$.

Si consideramos a una superficie cerrada como una región regular sin frontera, en la fórmula no hay contribucuón por la frontera ni por ángulos exteriores obteniendo el siguiente corolario.

Corolario 1 (Teorema de Gauss-Bonnet(do Carmo, 1994, §4-5)). Sea S una superficie cerrada, conexa y orientada. Entonces

$$
\iint_{S} K \mathrm{dvol}=2 \pi \chi(S)
$$

con $\chi(S)$ la característica de Euler-Poincaré.

Este teorema relaciona fuertemente la geometría y topología de la superficie. Por ejemplo, si la curvatura $K$ de $S$ es estrictamente positiva, entonces la integral es estrictamente positiva, lo que implica que $\chi(S)>0$. Como vimos, la esfera es la única superficie cerrada orientada 
con característica de Euler-Poincaré positiva. Por lo tanto, la curvatura impone restricciones a la topología de la superficie.

Ahora consideremos un triángulo geodésico $T$ en una superficie $S$, es decir, una región simple con sólo tres vértices, tal que los arcos regulares de su frontera sean geodésicas de $S$. Como una región simple es homeomorfa a un disco, tenemos que $\chi(T)=1$ y como la frontera está formada por geodésicas, la curvatura geodésica es cero, por lo tanto, en la fórmula la primera integral es cero. Expresando a los ángulos exteriores en términos de los ángulos interiores obtenemos.

Corolario 2. Sea T un triángulo geodésico en una superficie orientada S. Entonces

$$
\iint_{R} K \mathrm{dvol}=\eta_{1}+\eta_{2}+\eta_{3}-\pi,
$$

donde $\eta_{i}, i=1,2,3$, son los ángulos interiores de $T$.

Si $S$ es el plano euclidiano, $T$ es un triángulo euclidiano, cuyos lados son segmentos de recta (geodésicas en el plano), tenemos $K=0$, la integral es cero y obtenemos el conocido resultado de geometría euclidiana que la suma de los ángulos interiores de $T$ es igual a $\pi$. Si $S$ es la esfera unitaria, $T$ es un triángulo esférico, cuyos lados son segmentos de círculos máximos, tenemos $K=1$, la integral es estrictamente positiva y obtenemos que la suma de los ángulos interiores de $T$ es mayor a $\pi$, esto corresponde a la geometría elíptica. Si $S$ es el plano hiperbólico, tenemos $K=-1$, la integral es estrictamente negativa y obtenemos que la suma de los ángulos interiores de $T$ es menor a $\pi$, esto corresponde a la geometría hiperbólica.

Un espacio de Alexandrof $(X, d)$ es un espacio métricos geodésico con una noción sintética de curvatura acotada inferiormente, la cual se define comparando triángulos geodésicos en $X$ con triángulos euclidianos, elípticos o hiperbólicos. Los espacios de Alexandrof es un área muy activa de investigación actual, para saber más al respecto recomiendo el artículo (Moguel, 2021).

En 1944 Chern (Chern, 1944) generalizó el Teorema de Gauss-Bonnet para variedades diferenciables cerradas orientadas de dimensión $2 n$, expresando la característica de EulerPoincaré como la integral de cierto polinomio de su forma de curvatura.

\subsection{Superficies mínimas}

Una superficie $S$ es mínima si localmente minimiza su área. Una superficie $S$ es mínima sí y sólo sí su curvatura media es cero $(H=0)$ (do Carmo, 1994, §3-5). Ejemplos de superficies mínimas son las formadas por películas de jabón como la mostrada en la Figura 201100. 


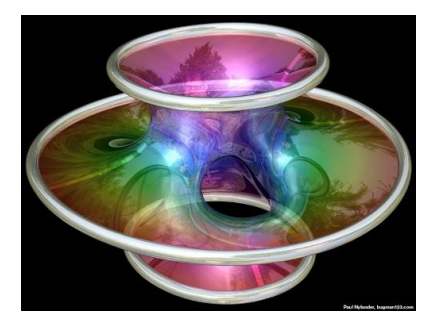

Figura 20: Superficie mínima de Costa.

Si el lector desea saber más sobre las superficies mínimas recomiendo el artículo (GalazGarcía, 2004).

\section{Regresando a la motivación}

Regresando a la motivación inicial del artículo, dado un plano $P$ en $\mathbb{R}^{3}$ y un punto $p$ en $P$, si tomamos cualquier otro plano $Q$ perpendicular a $P$ que pase por $p$, tenemos que la intersección de $P$ y $Q$ es una recta que pasa por $p$. Entonces todas las curvaturas normales de $P$ en $p$ son cero, y por lo tanto, la curvatura Gaussiana de un plano en todo punto es cero.

Por otro lado, consideremos la esfera unitaria $\mathbb{S}^{2}$ en $\mathbb{R}^{3}$ y un punto $p \in \mathbb{S}^{2}$. Si tomamos un plano $Q$ ortogonal a la esfera que pase por $p$, la intersección de $Q$ con $\mathbb{S}^{2}$ es un círculo máximo, el cual tiene curvatura 1 . Entonces, todas las curvaturas normales de $\mathbb{S}^{2}$ en $p$ son 1 y por lo tanto, la curvatura Gaussiana de la esfera es 1.

Como el plano y la esfera tienen diferente curvatura Gaussiana no podemos obtener un pedazo de esfera sin doblar, estirar o cortar una hoja de papel. Es por esto, que no podemos obtener un mapa perfecto.

\section{Para saber más}

Al lector interesado en saber más sobre las diferentes nociones de curvatura y de geometría Riemanniana en general, recomiendo el artículo (Río, 2002) y el libro (Muñoz, 2009). Para un estudio más formal, recomiendo los textos clásicos (do Carmo, 1992, 1994) y para una visión panorámica de la geometría Riemanniana el libro (Berger, 2003). 


\section{Notas}

1. Imagen tomada de http://www.grad.hr/itproject_math/Links/sonja/gausseng/ehpp/ehpp.htm1.

2. Imagen tomada de https://www.researchgate.net/figure/THE-GAUSS-MAP-By-a-direct-application-of-a-classical-result-of-Linear-Algebra-which_ fig1_330958425.

3. Imágen tomada de https://plus.maths.org/content/get-grips-mobius-strip.

4. Imágen tomada de https://plus.maths.org/content/imaging-maths-inside-klein-bottle.

5. Imágen tomada de https://math.stackexchange.com/questions/2634214/visualising-pi-2s2-and-pi-2-mathbbrp2.

6. Imágen tomada de http://www.supermath.info/ZooofMathematicalCreatures.htm.

7. Imágenes tomadas de http://www.earthdayenergyfast.org/IMAGES/earth.jpg http://www.navworld.com/navcerebrations/Toroidal.htm y http://www. ehu.es/ mtwmastm/CD0506.pdf.

8. Imágen tomada de http://www. cnmat.berkeley. edu/Research/AES2006/speakerarray/icosaspeakers.htm

9. Imágen tomada de http://www.math.cornell.edu/ mec/2003-2004/geometry/torii/torii.html.

10. Imágen tomada de http://bugman123.com/MinimalSurfaces/Costa-large.jpg. 


\section{Referencias}

Berger, M. (2003). A panoramic view of Riemannian geometry. Springer-Verlag, Berlin.

Chern, S.-S. (1944). A simple intrinsic proof of the gauss-bonnet formula for closed riemannian manifolds. Annals of Mathematics, 45(4):747-752.

do Carmo, M. (1992). Riemannian Geometry. Birkhauser.

do Carmo, M. P. (1994). Geometría Diferencial de Curvas y Superficies. Alianza Editorial.

Doyle, P. H. and Moran, D. A. (1968). A short proof that compact 2-manifolds can be triangulated. Invent. Math., 5:160-162.

Galaz-García, F. (2004). Superficies minimales. Miscelanea Matemática, 39:31-38.

Moguel, M. C. (2021). Espacios de Alexandrov y el problema de Erdös-Perelman. Miscelanea Matemática, 71:63-81.

Muñoz, J. L. (2009). Riemann: una nueva visión de la geometría. NIVOLA.

Naveira, A. M. (2007). L curvatura de riemann a través de la historia. Miscelanea Matemática, 44:29-52.

Osserman, R. (1990). Curvature in the eighties. Amer. Math. Monthly, 97(8):731-756.

Palmas, O. (2020). Todo cabe en un jarrito: una invitación a la teoría de inmersiones isométricas. Miscelanea Matemática, 70:59-75.

Río, E. G. (2002). Una introducción a la curvatura.

S. William Massey (1967). Algebraic Topology: an introduction. Springer Verlag.

Simanca, S. R. (2002). Geodésicas y curvatura: una introducción elemental. Miscelanea Matemática, 35:17-40.

Spivak, M. (1979). A Comprehensive Introduction to Differential Geometry, volume II. Houston, Texas (U.S.A.), second edition.

Como citar este artículo: Cisneros Molina, J. L. Un recorrido por el concepto de curvatura. SAHUARUS. REVISTA ELECTRÓNICA DE MATEMÁTICAS. ISSN: 2448-5365, 5(2), 1-27. https://doi.org/10.36788/sah.v5i2.120 